problems. The EQ-5D-3L was available in self-complete version, and proxy version for care-givers of patients mentally or physically incapable of reporting. In the proxy version, caregivers reported the HRQoL for both patients and themselves. A reverse crosswalk algorithm was used to generate five-level (EQ-5D-5L) utility scores from EQ-5D-3L profiles with reference to the Hong Kong value set, to allow comparison with the general population. The utility score ranges from 0 (death) to 1 (perfect health), with negative values indicating worsethan-death health states.

Results During the study period, 284 valid and independent responses were collected, including 165 patients from selfcomplete version and 119 patients and their care-givers from proxy version. The median age of patients and care-givers participated were 32.1 and 42.9 , respectively. The mean utility score of patients and care-givers were 0.5207 and 0.7992 , both significantly lower than that of the normal population, $0.9186(p<0.0001) .77(65 \%)$ of the 119 patients from the proxy version were below 18 years old, with a mean utility score of 0.4504 . Among all 284 patients, patients able to selfreport had a higher mean utility score, 0.5753, than patients requiring a care-giver for reporting, 0.4449. Patients requiring a proxy reported more severe problems in self-care and usual activities dimensions, while their care-givers reported more problems in anxiety/depression dimension. Overall, only $16 \%$ of patients and 29\% of care-givers had Level 1 in all five dimensions. In contrast, five (3\%) patients in self-complete version and $25(21 \%)$ patients in proxy version were reported with negative utility scores, in which two had Level 3 in all five dimensions. Out of the 30 patients with negative utility scores, $93 \%$ had no self-care ability, $87 \%$ were unable to perform usual activities and $67 \%$ were confined to bed.

Conclusions This is the first study in Asia to demonstrate that $\mathrm{RD}$ as a group has a negative impact on the HRQoL of both patients and care-givers. It illustrated the impact of RDs on different aspects of quality of life, which warrants exceptional care from policy makers and the society.

\section{ESTABLISHING NATIONWIDE STANDARDISED WORKPLACE-BASED LEARNING FOR PAEDIATRIC DOCTORS IN MYANMAR - A PILOT STUDY}

Nilar Lwin, Khin Nyo Thein, Ei Phyu Lwin, Ian Maconochie, Marcus Wootton, Aye Han. Myanmar

\subsection{6/bmjpo-2021-RCPCH.157}

Background The Myanmar Paediatric Society and the Royal College of Paediatrics and Child Health collaborated to set up a nationwide workplace-based learning and assessment strategy for MSc postgraduate paediatric doctors in Myanmar.

Objectives A Myanmar/UK joint working group created an Assessment portfolio and supported 40 supervisors and 170 students in its completion. The portfolio contained workplacebased practice learning (WPBL) approaches, including Directly Observed Procedures, Case-Based Discussions and the Handover Assessment Tool.

Postgraduate students (PPS) were asked to complete their portfolios during their clinical placements (in a range of Myanmar hospitals) with support and feedback from their clinical supervisors.
Methods Between August 2018 and December 2020 a twophase pilot was undertaken; phase 1 supported all second-year postgraduate students $(n=85)$, phase 2 continued with phase 1 year 2 group going into year 3, while also supporting the new year 2 cohort (another 85 PPS). Remote support was provided by senior Myanmar, UK paediatricians and educationalists throughout.

At phase 1 and 2 completion, all portfolios were collected, anonymised and assessed by senior Myanmar paediatricians affiliated to all Myanmar Medical Universities. Using a standardised rubric, including a 1-5 Likert scale, reviewers assessed the completeness of the portfolio and the quality of the feedback from the supervisor to the student.

Results Phase 1 portfolio results are presented, as at the time of submission phase 2 portfolios were being analysed, concluding in February 2021.

Overall completeness and quality scores

In the case of the portfolios, we found that more than. $69 \%$ had at least $50 \%$ of their portfolio sections completed. When we audited the supervisor feedback quality we found that $74 \%$ of the total were scored at 3 or above, adjudged to be at least a 'Fair attempt to comment on candidate performance and provide recommendations'.

In the case of Mini-CEX - Case selection there was a relatively even distribution of patient presentations selected by the postgraduate students in Mini-Cex showing a broad range of detailed clinical knowledge, which can be built on in the workplace through practical support.

\begin{tabular}{ll} 
Abstract 283 Table 1 & \\
\hline $\begin{array}{l}\text { Mini-Cex case selection } \\
\text { (case chose by trainees) }\end{array}$ & $\begin{array}{l}\text { The proportion of the cohort } \\
\text { (n85) }\end{array}$ \\
\hline Acute Respiratory Illness & $29 \%$ \\
Jaundice & $29 \%$ \\
Nephrotic Syndrome & $19 \%$ \\
Dengue Haemorrhagic Fever & $13 \%$ \\
Congenital Heart Disease & $6 \%$ \\
Pyrexia of Unknown Origin & $4 \%$ \\
\hline
\end{tabular}

Conclusions The pilot demonstrated the introduction of a standardised programme of non-didactic WBPL to support greater standardisation of practice across Myanmar.

Phase 1 demonstrated the importance of linking clinical practice to academic curricula, and setting up structures to support supervisors and PPS to deliver WBPL and constructive feedback.

The pilot helped to develop a cadre of supervisors able to support the enhancement of clinical learning within hospitals as sites of improved professional development.

There is further scope to explore the application of the methodologies of WBPL to assess and support wider clinician skills. Improving hospital systems to develop these skills can have a positive effect on the whole hospital system. For example, encouraging better leadership, governance, communication and teaching among paediatricians, will have wider positive implications for the care of children. 